\title{
THE OPTICAL PROPERTIES, DENSITIES, AND SOLUBIL- ITIES OF THE NORMAL FORMATES OF SOME METALS OF GROUP II OF THE PERIODIC SYSTEM
}

\author{
By F. W. Ashton, D. F. Houston, and C. P. Saylor
}

\section{ABSTRACT}

This paper presents data on the optical properties, densities, and solubilities in water from 0 to $100^{\circ} \mathrm{C}$. of the normal crystalline formates of calcium, strontium, barium, magnesium, zinc, and cadmium. The optical properties determined are the refractive indices, optic axial angle, elongation, interference figure, and dispersion. The crystal system and habit are also given.

The literature on previous work is discussed in connection with the present results. The system water-magnesium formate dihydrate was investigated and found to have a eutectic temperature of $-5.05^{\circ} \mathrm{C} . \pm 0.03$ at a composition corresponding to $14.0 \mathrm{~g} \pm 0.1$ of anhydrous salt per $100 \mathrm{~g}$ of water.

The transition temperature of strontium formate dihydrate to anhydrous formate was found to be $72.0^{\circ}$ to $72.5^{\circ} \mathrm{C}$. and that of cadmium formate dihydrate to anhydrous formate to be $66.0^{\circ}$ to $66.5^{\circ} \mathrm{C}$.

Harris's adaptation of Dühring's relation is applied to these salts, with cesium chlorate as a reference salt.

\section{CONTENTS}

II. Previous work

III. Materials_._. 235

1. Calcium formate...... 235

2. Strontium formate. 235

3. Barium formate.

4. Magnesium formate...... 236

5. Zinc formate.

6. Cadmium formate...... 237

IV. Optical properties_._.

V. Densities__._._.

1. Apparatus and procedure. 242

2. Experimental results

3. Discussion of results

VI. Solubilities._.

1. Apparatus and procedure

2. Investigation of magnesium formate 246

3. Experimental results

4. Discussion of results 250

5. Transition points

VII. Harris's adaptation of Dühring's relation.

VIII. Acknowledgment.

IX. Bibliography

\section{INTRODUCTION}

The formation of distinctive types or forms of crystals under given conditions has long been used by the organic chemist as a means of identifying certain classes of compounds, and most chemists have, 
at some time or other, used a simple or compound microscope for the examination of their materials. The use of the polarizing microscope in the identification of chemical compounds is becoming more widespread as the convenience and economy of time which may be so obtained are recognized. Its less prevalent use for organic materials is largely due, no doubt, to the fact that there are no extensive tables of optical properties of pure organic compounds like those available for minerals (1) ${ }^{1}$ and inorganic compounds (2). Such tables are necessary for the ready recognition and differentiation of these substances.

To encourage the use of so valuable a tool, it is desirable that there be determined, for series of organic compounds, accurate data which may serve as a source of reference for the organic chemist. The data should consist of those properties which would be of use in the microscopical identification of compounds-the optical character, the refractive index or indices, the optic axial angle, and the orientation of the principal optical directions with respect to those crystallographic directions which can be recognized. Such other crystallographic properties as the crystal system, crystal habit, and most commonly occurring form of crystal should also be given. Several articles have recently been published (3) which contain data of this type, and give references to the earlier work on this subject.

In this paper there will be included data on density and solubility also, since they are frequently useful in the identification of pure compounds.

The choice of the present subject for an initial project was governed by several conditions. At the inception of the work it was realized that it would be necessary to develop methods for the preparation of crystalline material of a sufficient degree of purity and in a form suitable for use with a microscope. It was also necessary to find more satisfactory immersion media for most of the organic compounds, since many of them are more or less soluble in the ordinary immersion liquids. It was decided, therefore, to begin with some of the simpler and more readily prepared compounds which could be investigated with the usual refractive index liquids. The formates of the elements of group II of the periodic system present such a series. Determination of the optical properties of these compounds, furthermore, forms a first step toward the preparation of tables of properties of the crystalline metallic compounds of the fatty acid series, and another possible method of identification of its members. Moreover, this group of formates is one for which some data exist, and the present work thus forms a check on previous results and supplies information hitherto lacking. This paper, therefore, presents the results of a study of the normal crystalline formates of calcium, strontium, barium, magnesium, zinc, and cadmium. No data are given for beryllium or mercury formate as no method has yet been found by which suitable crystalline material can be obtained.

\section{PREVIOUS WORK}

A survey of the literature of these compounds shows that data on optical properties, density, and solubility in water are often in disagreement, or are isolated or entirely lacking.

\footnotetext{
1 Figures in parentheses here and elsewhere in the text relate to the reference numbers given in the bibliography at the end of this paper.
} 
Crystallographic descriptions of anhydrous calcium, strontium, and barium formates, and of the strontium, zinc, and cadmium formate dihydrates have been compiled by Groth (4). No crystallographic descriptions of magnesium formate or of anhydrous cadmium formate have been found. Determinations of the optical properties of anhydrous calcium formate, of strontium formate dihydrate, and of barium formate are given by Schrauf (5), and of anhydrous strontium formate by Plathan (6).

Schröder (7) gives values for the densities of the formates of calcium, strontium, barium, zinc, and cadmium, and Clarke (8) reports values for barium, cadmium, and zinc formates. Incidental to his crystallographic studies, Plathan (6) reports density determinations on calcium, strontium, and barium formates; Wagner (9) gives data on calcium and strontium formates, and Alexatt (10) gives the density of strontium formate. The densities of calcium, strontium, and barium formates have also been calculated from X-ray data by Nitta (11).

Determinations of the solubilities of the formates of calcium, strontium, and barium are reported by Stanley (12); of calcium and barium formates by Krasnicki (13); and of calcium formate by Lumsden (14). No values were found for the other salts except the statement by Souchay and Grolle (15) that magnesium formate is soluble in 13 parts of water.

\section{MATERIALS}

\section{CALCIUM FORMATE}

The calcium formate used in this work was obtained from a commercial source. The salt, which is anhydrous, was recrystallized twice from aqueous solution, dried at $125^{\circ} \mathrm{C}$., and stored in a desiccator. The recrystallization served to remove insoluble matter containing iron. As was done with all the salts of this series except magnesium and zinc formates, total metal was determined by the following method: A weighed amount of the formate, contained in a porcelain crucible, was treated with dilute sulphuric acid. The solution was concentrated by evaporation, the acid driven off, and the residue ignited to dull redness, cooled, and weighed as sulphate. Combined strontium and barium were determined by the etheralcohol separation of the nitrates (16), which gives slightly high results. The material contains a slight amount of magnesium $(<0.01$ percent).

Calculated: $\mathrm{Ca}$ from $\mathrm{Ca}(\mathrm{HCOO})_{2}=30.80$ percent.

Found: Metals from sulphates, calculated as $\mathrm{Ca}=30.89,30.85$ percent.

Strontium and barium, calculated as $\mathrm{Ba}=<0.05$ percent.

\section{STRONTIUM FORMATE}

Strontium formate was prepared by adding strontium hydroxide to an excess of formic acid. It crystallizes as the dihydrate at ordinary temperatures and as the anhydrous salt above $70^{\circ} \mathrm{C}$. The material was recrystallized in three fractions as the dihydrate, and the first fraction again recrystallized. It was then dried at $125^{\circ} \mathrm{C}$. and analyzed. A portion was later recrystallized from a slightly acid solution at $80^{\circ}$ to $85^{\circ} \mathrm{C}$. and well-formed crystals were obtained for optical investigation. The dihydrate was made by a recrystallization at room temperature of the analyzed material. 
Calcium was determined by the ether-alcohol method (17), and barium in the residual material was determined by separation as dichromate (18). Total metals were also determined as the sulphates, and strontium was precipitated as the sulphate from an alcoholic solution (19).

Calculated: $\mathrm{Sr}$ from $\mathrm{Sr}(\mathrm{HCOO})_{2}=49.33$ percent.

$\mathrm{H}_{2} \mathrm{O}$ from $\mathrm{Sr}(\mathrm{HCOO})_{2} .2 \mathrm{H}_{2} \mathrm{O}=16.82$ percent.

Found: Metals from sulphates, calculated as $\mathrm{Sr}=49.33,49.35$ percent.

$\mathrm{Sr}$ from alcohol-precipitated sulphates $=49.32$ percent.

$\mathrm{Ca}$ from sulphate, calculated as metal $=0.02$ percent.

Ba from dichromate, calculated as metal $=<0.01$ percent.

$\mathrm{H}_{2} \mathrm{O}$ from dihydrate $=16.86$ percent.

\section{BARIUM FORMATE}

Barium formate, which is anhydrous, was obtained from a commercial source. It was recrystallized twice from aqueous solution, dried at $125^{\circ}$ C., and stored in a desiccator. The crystallizations removed traces of iron and insoluble matter. Calcium and strontium were determined by the ether-hydrochloric acid separation of the chlorides (20), and total metal was determined as sulphate.

Calculated: $\mathrm{Ba}$ from $\mathrm{Ba}(\mathrm{HCOO})_{2}=60.41$ percent.

Found: Metals from sulphates, calculated as $\mathrm{Ba}=60.32,60.35$, 60.33 percent.

$\mathrm{Ca}$ and Sr from chlorides $=<0.05$ percent.

A check sample was made in the laboratory by adding purified barium carbonate to an excess of formic acid. The carbonate was prepared from barium chloride (originally containing 0.05 percent of calcium and strontium) which had been twice extracted by means of ether-alcohol with an intervening fractional crystallization from aqueous solution. This purified chloride was treated with ammonium carbonate of high purity, and the resultant barium carbonate thoroughly washed. The barium formate from this preparation was fractionally crystallized, and was found to have the same optical properties as the previous preparation.

\section{MAGNESIUM FORMATE}

The magnesium formate was prepared by adding basic magnesium carbonate, containing about 0.02 percent each of calcium and iron, to an excess of formic acid. It was purified, as the stable dihydrate, by recrystallization from an aqueous solution containing a small amount of formic acid to prevent the formation of basic salts. The magnesium content was determined by precipitation and ignition to the pyrophosphate, and also by ignition to the oxide; the latter value more exactly represents total metals as oxides. The moisture determinations are not dependable, since there is a tendency to form a basic salt and liberate acid when driving off moisture. This in turn gives rise to somewhat high values for magnesium in the dehydrated salt. The tendency to lose acid is further discussed in connection with measurements of the density of this material.

Calculated: $\mathrm{Mg}$ from $\mathrm{Mg}(\mathrm{HCOO})_{2}=21.27$ percent.

$\mathrm{H}_{2} \mathrm{O}$ from $\mathrm{Mg}(\mathrm{HCOO})_{2} .2 \mathrm{H}_{2} \mathrm{O}=23.95$ percent.

Found: $\mathrm{Mg}$ weighed as $\mathrm{Mg}_{2} \mathrm{P}_{2} \mathrm{O}_{7}=21.37,21.43$ percent.

Metals from oxides, calculated as $\mathrm{Mg}=21.39$ percent.

$\mathrm{H}_{2} \mathrm{O}$ from dihydrate $=23.52,24.99$ percent. 


\section{ZINC FORMATE}

Zinc formate, which crystallizes as the dihydrate, was prepared and purified at the Naval Research Laboratory. It was further twice recrystallized from 1 percent formic acid solution, dried at $125^{\circ} \mathrm{C}$., and stored in a desiccator. The dihydrate was again formed by crystallizing the above salt at room temperature from a 1 percent formic acid solution by the addition of 95 percent alcohol.

Calculated: $\mathrm{Zn}$ from $\mathrm{Zn}(\mathrm{HCOO})_{2}=42.07$ percent.

$\mathrm{H}_{2} \mathrm{O}$ from $\mathrm{Zn}(\mathrm{HCOO})_{2} .2 \mathrm{H}_{2} \mathrm{O}=18.82$ percent.

Found: Metals from oxides, calculated as $\mathrm{Zn}=42.06,42.08$ percent. $\mathrm{H}_{2} \mathrm{O}$ from dihydrate $=18.78$ percent.

\section{CADMIUM FORMATE}

The cadmium formate, which crystallizes either as the dihydrate or the anhydrous salt, was obtained from a commercial source. Heating the solution on the steam bath gave a precipitate containing iron, which was filtered off. The salt was then twice recrystallized from a weak formic acid solution. The product was dried at $105^{\circ} \mathrm{C}$., stored in a desiccator, and analyzed. The dihydrate was then formed by recrystallization of the analyzed salt at room temperature from a 1 percent formic acid solution. The anhydrous salt was obtained by recrystallization of the analyzed salt from 1 percent formic acid solution at $70^{\circ}$ to $75^{\circ} \mathrm{C}$.

Calculated: $\mathrm{Cd}$ from $\mathrm{Cd}(\mathrm{HCOO})_{2}=55.52$ percent.

$$
\mathrm{H}_{2} \mathrm{O} \text { from } \mathrm{Cd}(\mathrm{HCOO})_{2} .2 \mathrm{H}_{2} \mathrm{O}=15.12 \text { percent. }
$$

Found: Metals as sulphates, calculated as $\mathrm{Cd}=55.47,55.50$ percent. $\mathrm{H}_{2} \mathrm{O}$ from dihydrate $=15.12$ percent.

\section{OPTICAL PROPERTIES}

The optical properties of this group of compounds were determined by the usual methods of optical petrography. ${ }^{2}$ According to these general methods, crystals or crystal fragments are immersed in liquids chosen from a series of which the refractive indexes vary progressively. Different liquids are tried until one is found with a refractive index as nearly as possible the same as that of the crystal when viewed by plane polarized light, vibrating parallel to a principal vibration direction in the crystal. Adjacent liquids in the series are finally mixed in order that the refractive index of the crystals may be determined more accurately. In this way three refractive indices at right angles to each other are determined for each substance.

In the present work the orientation of every crystal or crystal fragment upon which an index is based was checked by means of its interference figure. To determine the relation between the refractive indices of the immersion liquid and the crystals both the method of central and the method of oblique illumination were employed. When a crystal index was matched by a mixture of liquids, the refractive index of the liquid for sodium light was determined by readings with an Abbe refractometer. In the operation of matching, either mean white light or monochromatic light from a mercury vapor lamp, $\lambda=0.577-9 \mu$, was employed. Since the dispersion of the liquids is only slightly greater than that of the crystals, this procedure gives results which are correct for sodium light within the limit of

2 These methods can be found summarized in any manual of optical petrography; for example, Albert Johannsen, Manual of Petrographic Methods, John Wiley \& Sons, New York, 1918. See also reference 1. 
accuracy of the method of matching. The error in the determination of refractive indices is believed to be not greater than \pm 0.002 .

The optic axial angles of barium formate and strontium formate dihydrate were determined by universal stage methods (31) upon whole crystals grown directly upon the microscope slide where they were studied. The optic axial angle of anhydrous strontium formate was determined upon crystal fragments with an oil-immersion objective, N.A. 1.45, and the optic axial angles of magnesium formate, zinc formate, the two cadmium formates, and calcium formate were determined upon crystal fragments with a dry objective, N.A. 0.85. Determinations were made by interference figures upon fragments with the acute bisectrix approximately perpendicular to the stage of the microscope. The relation between polar distance in the interference figure and the obliquity of a ray was directly determined with an Abbe apertometer. From the corrected obliquities, $\rho_{1}$ and $\rho_{2}$, of the optic axes and the angle, $\theta$, between the two planes which contain the axis of the microscope and an optic axis, the optic axial angle, $2 V$, was calculated from the relation

$$
\cos 2 V=\cos \rho_{1} \cos \rho_{2}+\sin \rho_{1} \sin \rho_{2} \cos \theta .
$$

The data are summarized in table 1 , which is self-explanatory save for the following: In the last column drawings of crystals are exhibited in the positions in which they ordinarily occur when grown upon the microscope slide. To the right of each crystal is a key to its optical orientation. A stereographic projection indicates the directions of $\alpha, \beta, \gamma$, the optic axes, and the acute and obtuse bisectrices. Their points of emergence on the surface of the sphere of projection are represented by uniform symbols. Planes of optical symmetry are drawn as dotted lines, and directions which lie in the plane of the projection are indicated by full lines. In column 7 the faces of rhombic crystals have been described with reference to vibration directions rather than crystallographic directions, because the latter are of ten ambiguous under the microscope.

The refractive indices of anhydrous strontium formate differ from the values found by Plathan (6a) and those of barium formate differ from Schrauf's (5) more than can be explained by any probable error of observation. It is thought that the discrepancy is due to differences in the purity of the samples. In Schrauf's publication there is no record of any analysis having been performed. To describe his samples, Plathan employed the dubious phrase that they were "spectroscopically pure"! The analyses of the samples used in this work are given on page 236 . Samples of barium formate from two independent sources were employed and their optical properties were determined by separate observers with the following results:

\begin{tabular}{|c|c|c|c|}
\hline & $a$ & $\beta$ & $\gamma$ \\
\hline $\begin{array}{l}\mathrm{Ba}(\mathrm{HCOO})_{2} \text { purified from commercial formate } \\
\mathrm{Ba}(\mathrm{HCOO})_{2} \text { made from barium chloride. }\end{array}$ & $\begin{array}{l}1.567 \\
1.566+\end{array}$ & $\begin{array}{l}1.587 \\
1.587\end{array}$ & $\begin{array}{l}1.627 \\
1.628\end{array}$ \\
\hline
\end{tabular}

The values observed for strontium formate dihydrate agree with those of Schrauf, but those for the anhydrous salt differ sharply from Plathan's values. The difficulties in separating members of the alkaline-earth series still persist, but they were much less surmountable 35 years ago. For this reason it is plausible to suppose, particularly in the absence of detailed analyses, that some of these earlier salts were contaminated by other members of the isomorphous series. 


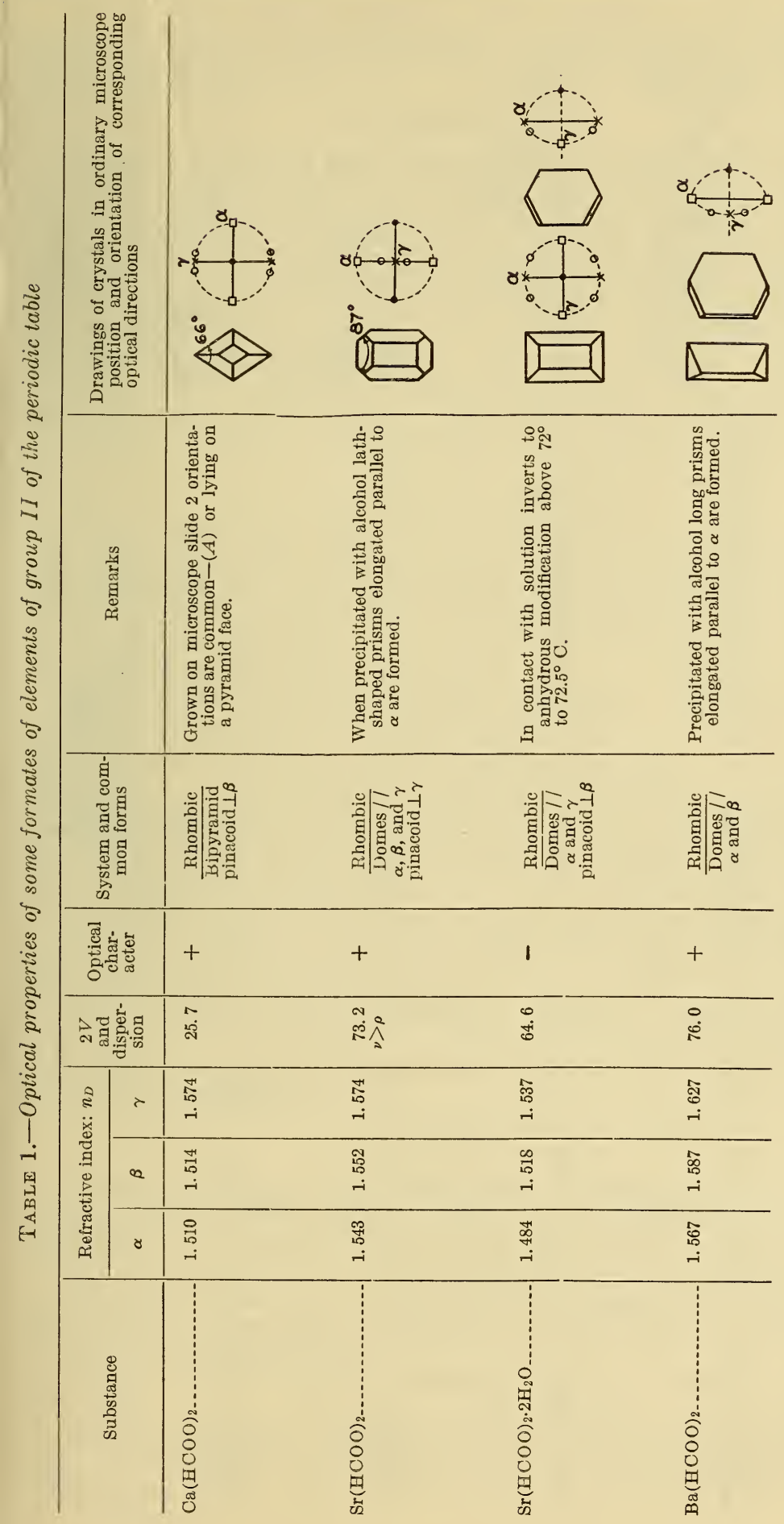




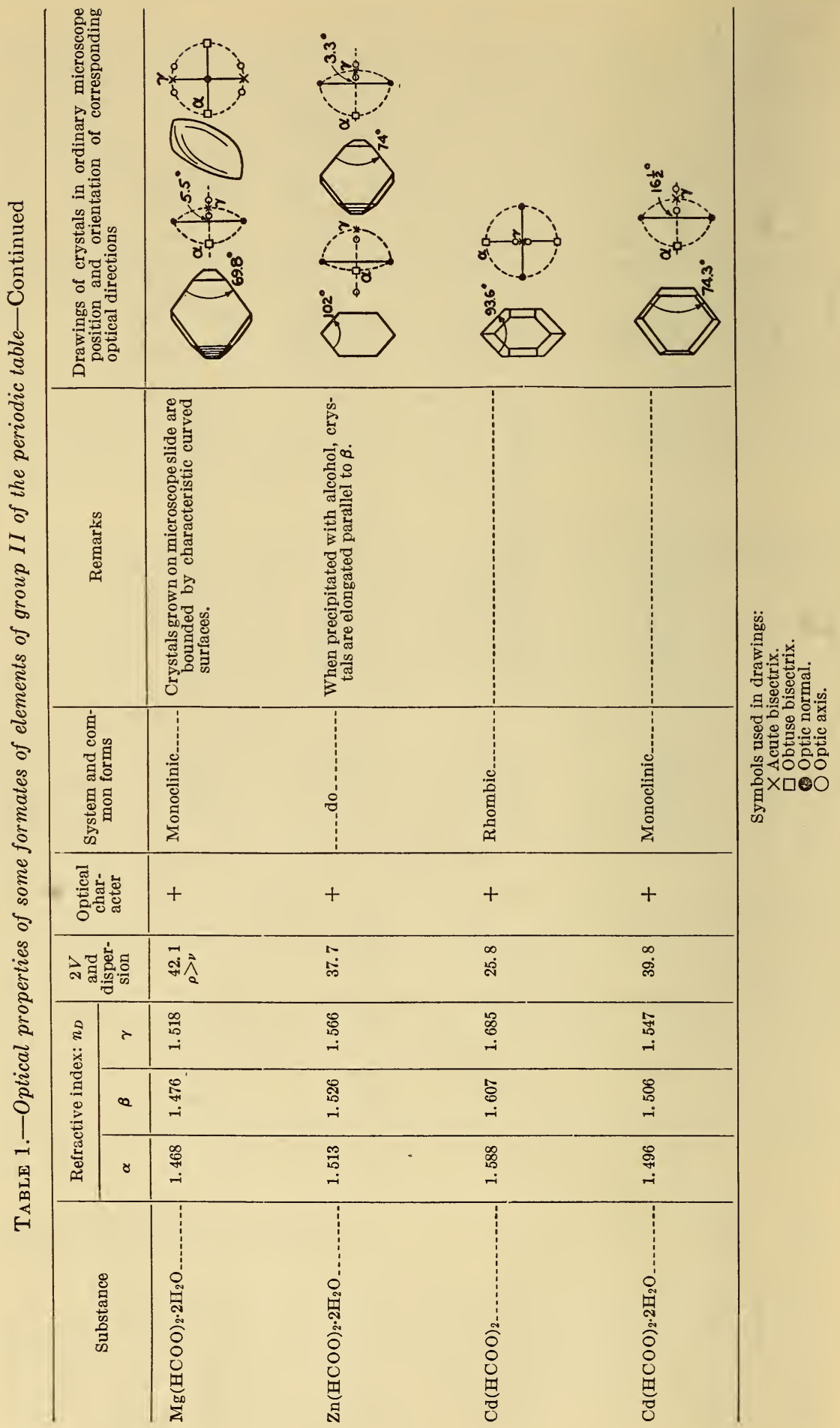


In the following description of the crystals of these formates, reference is made to the drawings in the last column of table 1 . For each substance the drawing to the left is indicated by $(A)$, that to the right by $(B)$.

\section{(a) CALCIUM FORMATE}

Crystal system and habit.-Rhombic. Bipyramidal crystals with pinacoid perpendicular to $\beta$. Grown upon a microscope slide they stand upon this pinacoid $(A)$ or upon a bipyramid face. When they stand upon the bipyramid face they exhibit the diamond-shaped, hexagonal, or triangular outlines characteristic of this form.

Refractive indices $\left(n_{D}\right) .-\alpha(=1.510)$ and $\gamma(=1.574)$ are exhibited by crystals $(A)$ grown upon the pinacoid face. $\beta(=1.514)$ could only be determined upon fragments.

Other optical properties.-Biaxial, positive. Optic axial angle $2 V=26^{\circ}$.

Interference figures.-Crystals like $(A)$ exhibit optic-normal figure.

(b) STRONTIUM FORMATE

Crystal system and habit.-Rhombic. Crystals were not grown on the microscope slide. There are three primary domes and a pinacoid perpendicular to $\gamma$. Crystals precipitated by addition of hot alcohol to a hot solution are lath-shaped and elongated parallel to $\alpha$.

Refractive indices $\left(n_{D}\right) .-\alpha(=1.543), \beta(=1.552)$, and $\gamma(=1.574)$ were all determined upon fragments.

Other optical properties.-Biaxial, positive. Optic axial angle $2 V=73^{\circ}$. Dispersion of the axial angle: $\nu>\rho$.

\section{(c) STRONTIUM FORMATE DIHYDRATE}

Crystal system and habit.-Rhombic. Principal forms are a pinacoid perpendicular to $\beta$ and domes parallel to $\alpha$ and $\gamma$. Grown upon a microscope slide they rest, as in $(A)$ upon the pinacoid, or upon the dome parallel to $\alpha$ as in $(B)$.

Refractive indices $\left(n_{D}\right) .-\alpha(=1.484)$ and $\gamma(=1.537)$ were determined on crystals like $(A)$ which rest upon the pinacoid. $\beta(=1.518)$ was determined on fragments.

Other opical properiies.-Biaxial, negative. Optic axial angle, $2 V=65^{\circ}$.

Interference figures.-Crystals like $(A)$ on a pinacoid face exhibit an optic-normal figure.

\section{(d) BARIUM FORMATE}

Crystal system and habit.-Rhombic. Crystals grown on a microscope slide rest upon a dome parallel to $\alpha$. Another form is a dome parallel to $\beta$. Precipitation with alcohol yields needles elongated parallel to $\alpha$.

Refractive indices $\left(n_{D}\right)-\alpha(=1.567)$ is parallel to the long direction of crystals as they grow on a slide. $\beta(=1.587)$ and $\gamma(=1.627)$ were determined on fragments.

Other optical properties.-Biaxial, positive. Optic axial angle, $2 V=76^{\circ}$.

Interference figure.-With an objective of numerical aperture 0.85, the acute bisectrix and principal plane of crystals like $(B)$ lie just outside the field. 
(e) MAGNESIUM FORMATE DIHYDRATE

Crystal system and habit.-Monoclinic. Crystals $(A)$ grown slowly are bounded by plane surfaces and have the same habit as zinc formate dihydrate. When grown on the microscope slide many of them are twinned and all of them are bounded by curved surfaces.

Refractive indices $\left(n_{D}\right) .-\alpha(=1.468)$ and $\gamma(=1.518)$ are commonly observed on the curved crystals $(B)$ grown on the slide. $\beta(=1.476)$ bisects the obtuse angle in crystals like $(A)$.

Other optical properties.-Biaxial, positive. Optic axial angle, $2 V=42^{\circ}$. Dispersion of the axial angle: $\rho>\nu$.

Interference figures. - Whole crystals like $(A)$, in table 1 , show an optic axis inclined $6^{\circ}$ from center.

\section{(f) ZINC FORMATE DIHYDRATE}

Crystal system and habit.-Monoclinic. When grown on a slide the crystals are as represented in table 1 . When precipitated by alcohol they are elongated parallel to $\beta$.

Refractive indices $\left(n_{D}\right) .-\alpha(=1.513)$ and $\gamma(=1.566)$ were determined on fragments. $\beta(=1.526)$ can be determined on nearly any whole crystal.

Other optical properties.-Biaxial, positive. Optic axial angle, $2 V=38^{\circ}$.

Interference figures.-Whole crystals like $(A)$ show an optic axis inclined $3^{\circ}$ from center.

\section{(g) CADMIUM FORMATE}

Crystal system and habit.-Rhombic. Principal forms are brachypinacoid, bipyramid, prism, and macropinacoid. The acute bisectrix is perpendicular to the brachypinacoid. $\beta$ is parallel to the crystallographic axis $\alpha$. Satisfactory crystals for the determination of the system are difficult to obtain. Massive groups and clusters of needles parallel to each other are common.

Refractive indices $\left(n_{D}\right)$.- All indices were determined upon irregular fragments. $\alpha=1.588, \beta=1.607, \gamma=1.685$.

Other optical properties.-Biaxial, positive. Optic axial angle, $2 V=26^{\circ}$.

\section{(h) CADMIUM FORMATE DIHYDRATE}

Crystal system and habit.-Monoclinic. Crystals grown on a slide have the same habit as zinc formate dihydrate. Grown in a beaker $(A)$ they have several additional forms.

Refractive indices $\left(n_{D}\right) .-\alpha=1.496, \beta=1.506, \gamma=1.547$.

Other optical properties.-Biaxial, positive. Optic axial angle, $2 V=40^{\circ}$.

Interference figures.-Whole crystals like $(A)$ exhibit an optic axis inclined $16^{\circ}$ from center.

\section{DENSITIES}

\section{APPARATUS AND PROCEDURE}

For the determination of density the pycnometer method was used. The pycnometer was of the type employed by Richards, Hall, and 
Mair (21) and is illustrated in figure 1. Each of the ground glass joints was given a broad bevel $(1: 5)$, finely ground, to insure reproducible settings. That this was achieved, though no lubricant was used, is shown by the fact that the weight of the pycnometer containing xylene and a salt, and adjusted to the volume mark at $20^{\circ} \mathrm{C}$., was reproducible to $0.2 \mathrm{mg}$.

For the measurements a temperature of $20.000^{\circ}$ C. \pm 0.005 was maintained by means of a large water bath, which is further described in connection with the solubility determinations.

The density was determined in all cases upon ground samples to prevent errors due to the presence of voids or inclusions of mother liquor. The salt, after weighing the sample in the pycnometer, was covered with the reference liquid and the apparatus placed in a vibrating chamber under reduced pressure to remove all entrapped air. The pycnometer was then filled and held at the desired temperature for an hour. The volume of liquid was then adjusted, and the pycnometer removed, dried, and allowed to stand in the balance case for one half hour before weighing.

The reference liquid used was xylene. It was purified and dried over sodium hydroxide, then over sodium wire, and finally distilled; the first and last portions were rejected. It was stored in a glass container and its density was determined just before the series of density determinations.

Slight evaporation of the reference liquid occurred, as no lubricant was used on the ground glass joints. This was evaluated and a correction made for it. In all weighings the pycnometer was counterpoised by a duplicate, and all weighings were corrected to vacuum.

\section{EXPERIMENTAL RESULTS}

The values obtained, together with the results of previous investigators for comparison, are given in table 2 .

TABLe 2.-Densities

\begin{tabular}{|c|c|c|c|c|c|}
\hline \multirow[b]{2}{*}{ Salt } & \multicolumn{3}{|c|}{ Previous investigations } & \multicolumn{2}{|c|}{ Present results } \\
\hline & $\begin{array}{l}\text { Calculated } \\
\text { from X-ray } \\
\text { data }\end{array}$ & $\begin{array}{l}\text { In crystallo- } \\
\text { graphic in- } \\
\text { vestigations }\end{array}$ & Pycnometric & Individual & $\begin{array}{l}\text { Weighted } \\
\text { average }\end{array}$ \\
\hline $\mathrm{Ca}(\mathrm{HCOO})_{2} \ldots$ & ${ }^{1} 2.02(11)$ & $\begin{array}{ll}2.015 & (6) \\
2.023 & (9)\end{array}$ & $\begin{array}{l}2.009(7 \mathrm{~b}) \\
2.021(7 \mathrm{a})\end{array}$ & $\begin{array}{r}2.0133 \\
2.0160\end{array}$ & 2.014 \\
\hline & 2. 71(11) & $\begin{array}{l}2.693 \quad(6) \\
2.693 \quad(9)\end{array}$ & $2.667(7 b)$ & $\begin{array}{l}2.6975 \\
2.6967 \\
2.6946\end{array}$ & 2. 696 \\
\hline $\mathrm{Sr}(\mathrm{HCOO})_{2 .} 2 \mathrm{H}_{2} \mathrm{O}$ & 2. 26(11) & $\begin{array}{l}2.2549(10) \\
2.259(9)\end{array}$ & $\begin{array}{l}2.240(7 \mathrm{~b}) \\
2.257(7 \mathrm{~b}) \\
2.234(7 \mathrm{~b}) \\
2.266(7 \mathrm{a}) \\
2.252(7 \mathrm{a})\end{array}$ & $\begin{array}{l}2.2467 \\
2.2445 \\
2.2481\end{array}$ & 2. 246 \\
\hline
\end{tabular}

1 Figures in parentheses refer to reference numbers in the bibliography at the end of this paper. 
TABle 2.-Densities-Continued

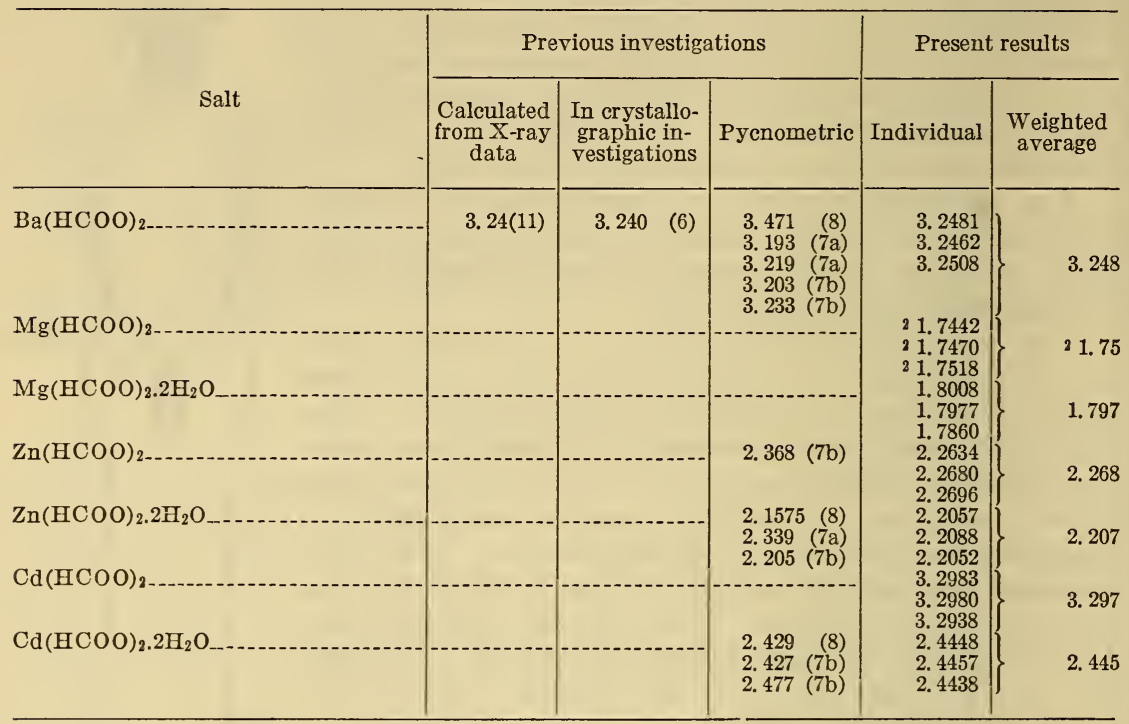

2 The accuracy of these values is questionable.

\section{DISCUSSION OF RESULTS}

Two sources of possible error must be considered in evaluating the present results, one arising from the material and one from the procedure.

The probability that impurities in the present samples affected the results is negligible, though the possibility of incomplete removal of entrapped air may well be considered. Though the magnitude of such an effect cannot be readily estimated of itself, the fact that it is not large may be assumed from the reproducibility of the results. Single low values occur in the cases of magnesium formate dihydrate, and anhydrous zinc and cadmium formates. These may be the results of incomplete removal of air, and are accordingly weighted one half the other results in computing the average.

In view of the limitations imposed by these causes it is believed that the weighted averages given in table 2 are accurate to \pm 0.002 except for anhydrous magnesium formate.

The result given for this latter salt represents the best values obtainable on the material dried at $110^{\circ} \mathrm{C}$. and under $5 \mathrm{~mm}$ pressure. Xylene was added at pressures of $3 \mathrm{~mm}$ and less to insure complete removal of entrapped air. This salt shows a very strong tendency to lose acid during dehydration, though Kendall and Adler state that it may be dried to constant weight at $110^{\circ} \mathrm{C}$. with negligible hydrolysis and loss of acid (22). An attempt to secure air-free anhydrous formate by heating it under xylene in an apparatus similar to that for determining the water content of oils (23) resulted in appreciable loss of acid and lower density of the remaining material.

The majority of previous density determinations on these materials have been made by Schröder (7). His results show considerable variation among the values for single salts, and consistently higher 
values for powdered material than for complete crystals. Though he indicates the materials to be of good quality, detailed information is generally lacking.

The values given from Clarke's laboratory (8) are said to be "the mean of several closely concordant estimations", and the specific gravity of cadmium formate is recorded as 2.429. Schröder, however, states (24) that the values found by Breen in Clarke's laboratory for this salt are 2.421 to 2.438 . Breen's value for barium formate is so high as to appear in error. No analyses are given for these salts.

The measurements made in connection with crystallographic investigations are in better agreement, though information concerning methods of preparation, and of purity, is usually not given.

The values obtained from X-ray investigations are essentially in agreement with the present findings.

\section{SOLUBILITIES}

\section{APPARATUS AND PRO. CEDURE}

The apparatus used in determining the solubility of these salts in water is shown in figure 2. It was constructed in such a manner that filtration could be carried out within the main solution, and was arranged for sampling by pressure, rather than suction, to prevent loss of solvent. The filter tube was immersed in the solution just before sampling so that any liquid in the tube would be near equilibrium conditions. The tube was, however, kept partially in the solubility chamber so that it would be

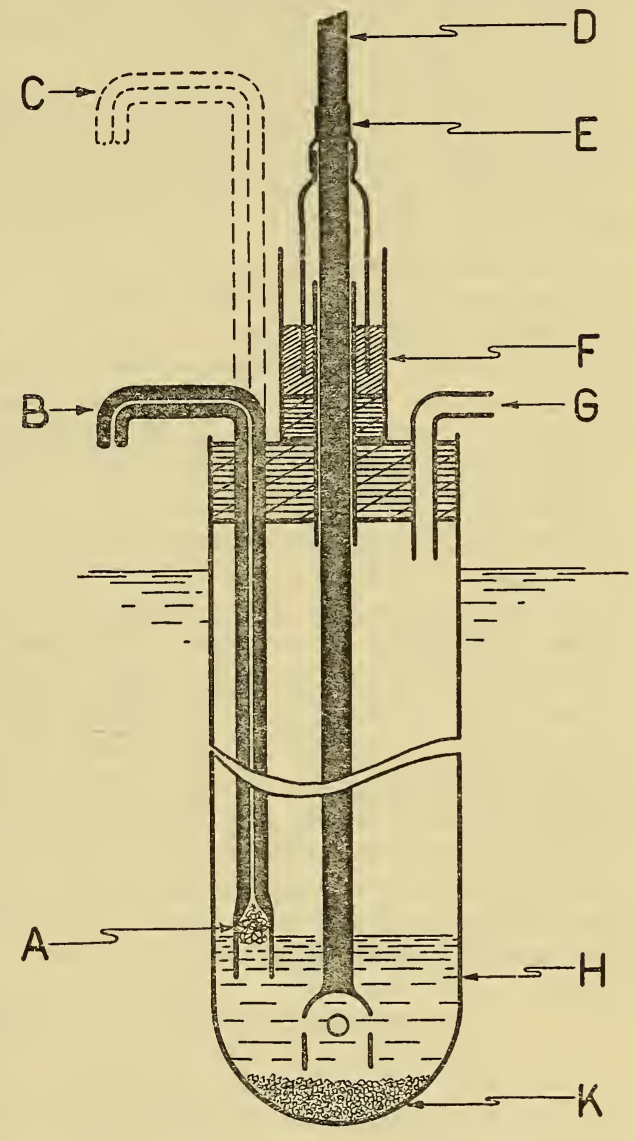

FIGURE 2.-Apparatus for solubility determinations.

$A$, filter; $B$, sample tube in sampling position; $C$, same in rest position; $D$, motor-driven stirrer; $E$, rubber connection; $F$, mercury seal; $\cdot G$, air inlet for sampling by pressure; $H$, saturated solution; $K$, excess salt. at approximately the correct temperature.

Solubilities at $0^{\circ} \mathrm{C}$. were determined with the chamber in a bath of melting ice.

At all other temperatures the solubility chamber was immersed in the constant-temperature water bath. This bath was contained in a well-insulated 16-liter earthenware jar, was covered with a layer of paraffin, and was fitted with a mechanical stirrer. For heating 
above room temperatures, the bath was equipped with knife-type heaters and electric lamps wired in parallel and actuated by a mercury regulator of the positive action type designed by H. J. Wing (25). Xylene was used as the expansion liquid, rather than toluene, to lessen evolution of gas at the higher temperatures. For temperatures below that of the room the bath was cooled by an immersed coil carrying tap water or ice water and compensated by the above heating and regulating units. At or near room temperature the control was within $\pm 0.005^{\circ} \mathrm{C}$., though at the extremes of the range it was not better than $\pm 0.05^{\circ} \mathrm{C}$.

The procedure was the same for all salts except magnesium formate. In this case difficulties were encountered which demanded further investigation, and the results are discussed separately.

In all but a few instances saturation equilibrium was approached from both directions. The solubility at any temperature was determined after adjusting the solution to that temperature from one approximately $10^{\circ} \mathrm{C}$. above or below. It was found that equilibrium was not surely attained in some cases, particularly at the lower temperatures, in less than 4 hours; accordingly, the procedure was developed of taking samples morning and evening. This gave periods of 6 to 18 hours. Both the solution and the bath were stirred continuously during the period of attainment of equilibrium.

The filter tube, after cleaning and drying, was fitted with a new cotton filter plug, introduced nearly to the solution, and allowed to come to temperature before immersion. Duplicate samples of 1 to $3 \mathrm{ml}$ were taken after the filter had been immersed a short while. A few milliliters of solution were first forced over to bring the tube to temperature and overcome any change in concentration caused by adsorption on the filter. A sample was next filtered into a weighed, clean, dry, weighing bottle which was immediately closed with a ground glass stopper. Several more milliliters were forced over and then a second sample was taken. The weighing bottles were allowed to come to room temperature and weighed. The solutions were evaporated in an oven heated to $125^{\circ} \mathrm{C}$., cooled in a desiccator, and the bottles reweighed. From the weight of anhydrous salt thus obtained, the solubility in grams per $100 \mathrm{~g}$ of water was calculated.

\section{INVESTIGATION OF MAGNESIUM FORMATE}

The same procedure for sampling was possible in the case of this salt, but attempts to evaporate the solutions led to decomposition. Check values could not be obtained. Accordingly the weighed solution was transferred to a porcelain crucible, evaporated to dryness, and the residue ignited to magnesium oxide. In this way concordant values were obtained on duplicate samples.

This procedure gave results which accurately determined the solubility at higher temperatures, but below $40^{\circ} \mathrm{C}$. gave too large an uncertainty. This was at first believed to be due to a change of hydrate in equilibrium, but analyses of the salt in contact with the saturated solution at various temperatures from $0^{\circ}$ to $75^{\circ} \mathrm{C}$. showed it to be the dihydrate in all cases.

In order to locate the lower portion of the solubility curve more accurately, the eutectic of the system $\mathrm{Mg}(\mathrm{HCOO})_{2}-\mathrm{H}_{2} \mathrm{O}$ was deter- 
mined. This was done by determining the freezing points of a series of solutions containing known graduated amounts of magnesium formate. Measurements of the freezing point of solutions containing: $5.7 \mathrm{~g}$ and less of magnesium formate per $100 \mathrm{~g}$ of water have been made by Calamé (26). The present measurements were made with solutions having concentrations greater than those used by Calamé. Determinations were made in the Beckmann type of apparatus, with constant stirring, and the temperatures were determined with a thermometer graduated in $0.1^{\circ} \mathrm{C}$. and calibrated at this Bureau. In this way the ice curve and the eutectic temperature of the system were located.

The upper, definite portion of the solubility curve was then extrapolated to meet the ice curve at the eutectic temperature, which gives a lower portion of the solubility curve within the limits found by the earlier procedure. The accuracy of this location is further

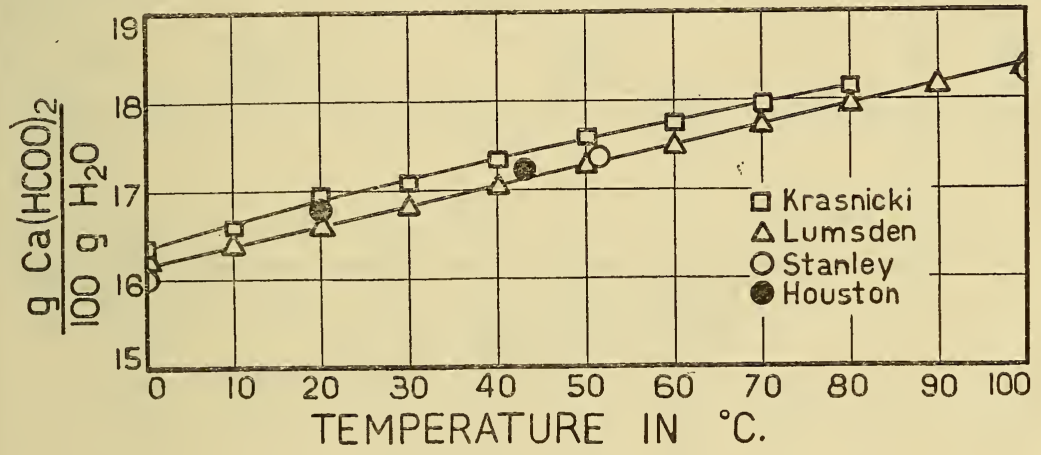

Figure 3.-Solubility of calcium formate.

indicated by the agreement obtained in the adapted Dühring's relation, which is presented in a following section.

\section{EXPERIMENTAL RESULTS}

The various investigations (12)(13)(14) of the solubility of calcium formate in water agree rather closely. This salt was accordingly investigated at two points to check the present method. The values, shown in figure 3 , are concordant with previous data.

The values obtained for the solubility of strontium formate are shown in figure 4, together with the results of Stanley (12) for ready comparison.

The two existing determinations of the solubility of barium formate (12)(13) are not at all in agreement. They are given in figure 5, together with the results of the present investigation.

No previous determinations of solubility were found for the formates of magnesium, zinc, or cadmium, except the statement (15) that magnesium formate is soluble in 13 parts of water. The solubilities found for zinc and cadmium formates are given in figure 6 , and the results of the investigation on magnesium formate in figure 7 .

The solubilities of the formates investigated are shown in table 3 at 10-degree intervals; the solubilities of strontium and cadmium formates are also shown at the transition points. 


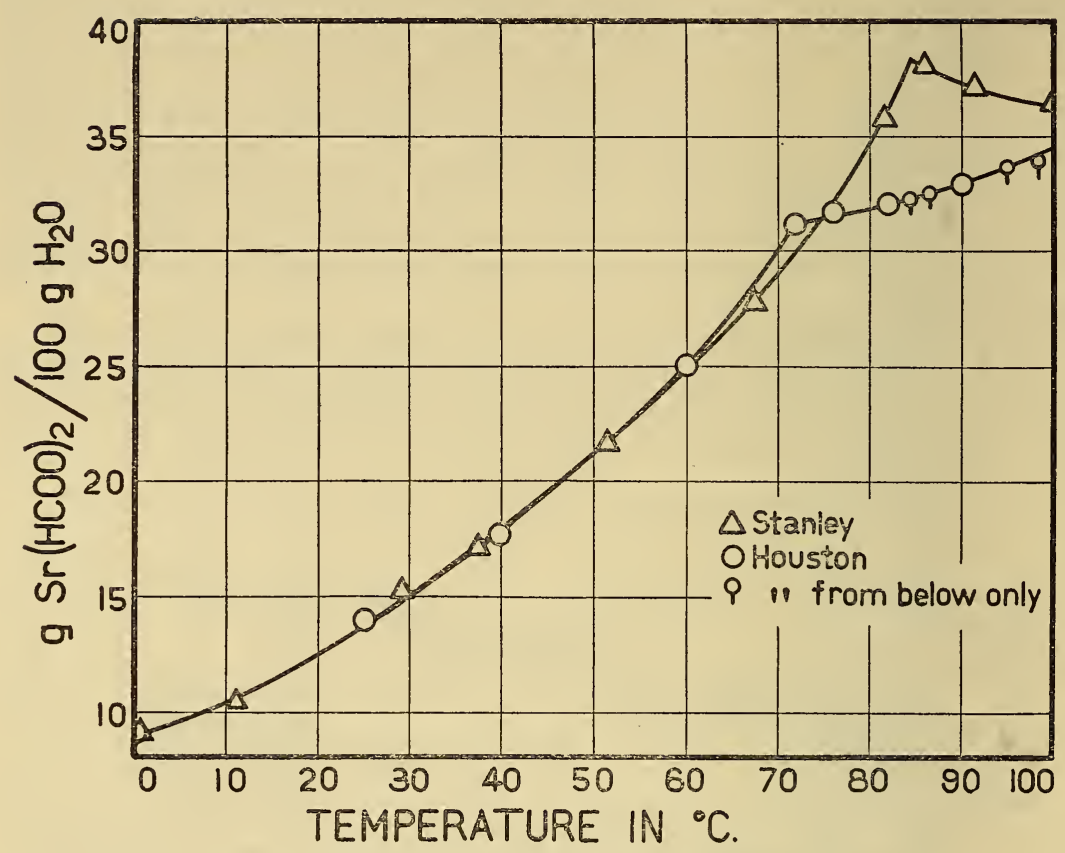

FIGURE 4.-Solubility of strontium formate.

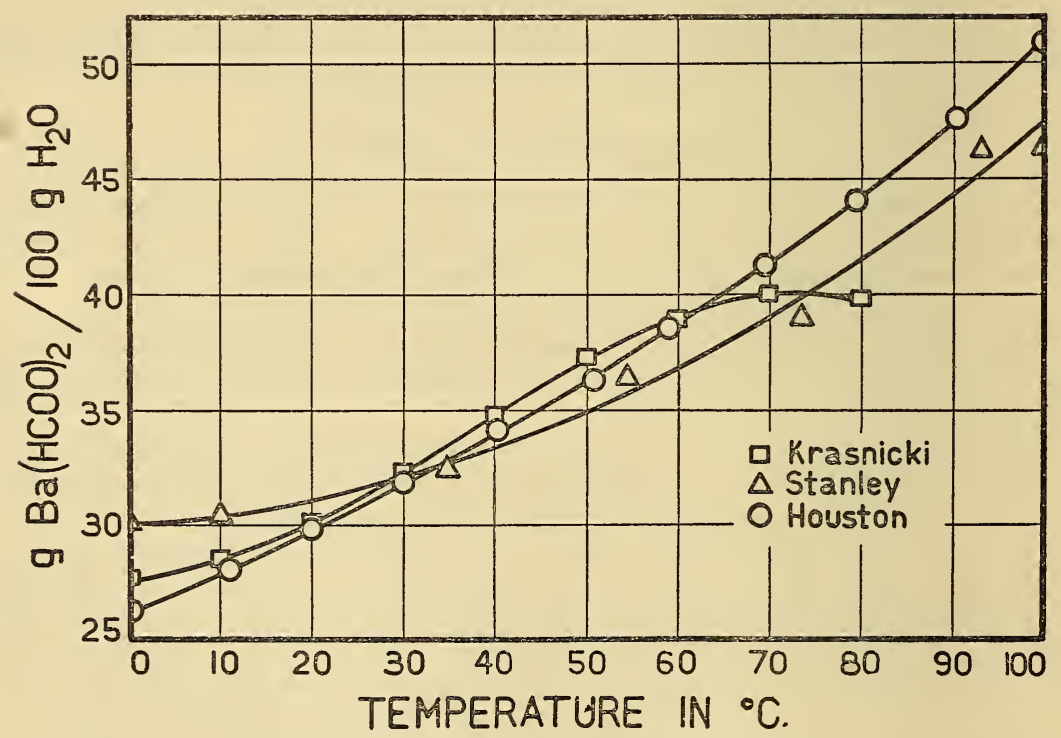

Figure 5.-Solubility of barium formate. 


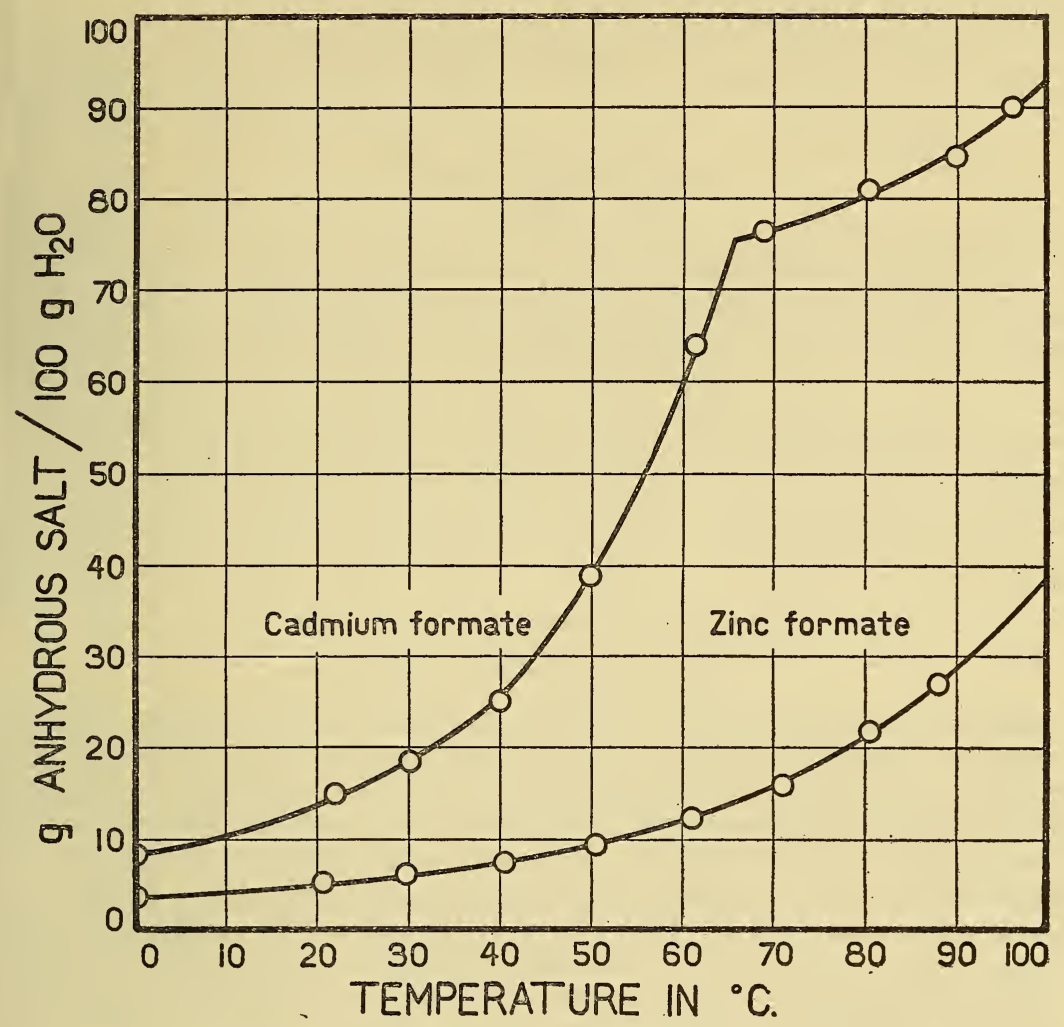

Figure 6.-Solubility of zinc"and"cadmium formates.

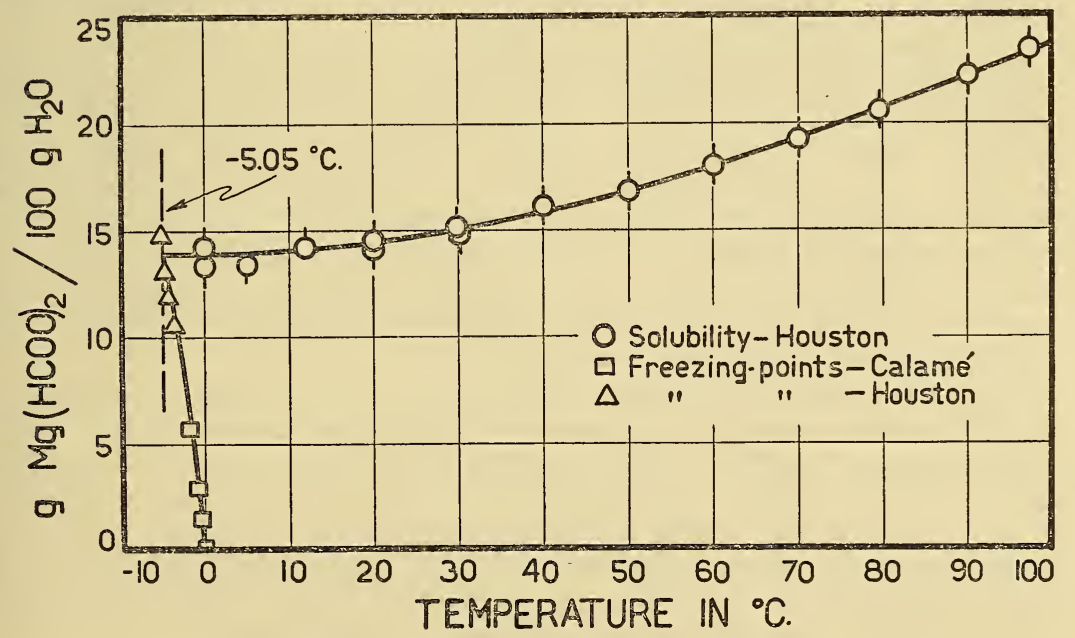

Figure 7.-Sysiem $\mathrm{Mg}(\mathrm{HCOO})_{2}-\mathrm{H}_{2} \mathrm{O}$. 
TABLE 3.-Solubilities

\begin{tabular}{|c|c|c|c|c|c|c|c|c|}
\hline \multirow[b]{2}{*}{ Temperature (in ${ }^{\circ} \mathrm{C}$.) } & \multicolumn{8}{|c|}{ Solubility in grams of anhydrous salt per $100 \mathrm{~g}$ of water } \\
\hline & 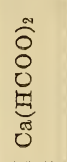 & 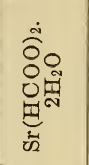 & 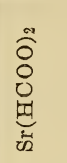 & 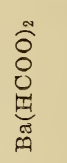 & 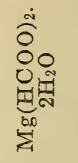 & 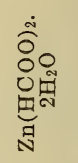 & 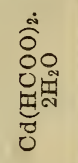 & 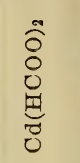 \\
\hline $\begin{array}{l}0 \\
10 \\
20 \\
30 \\
40\end{array}$ & $\begin{array}{l}16.2 \\
16.4 \\
16.6 \\
16.8 \\
17.0\end{array}$ & $\begin{array}{r}9.1 \\
10.6 \\
12.7 \\
15.2 \\
17.8\end{array}$ & 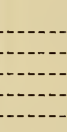 & $\begin{array}{l}26.2 \\
28.0 \\
29.9 \\
31.9 \\
34.0\end{array}$ & $\begin{array}{l}14.0 \\
14.1 \\
14.4 \\
15.0 \\
15.9\end{array}$ & $\begin{array}{l}3.7 \\
4.3 \\
5.2 \\
6.1 \\
7.4\end{array}$ & $\begin{array}{r}8.4 \\
11.1 \\
14.4 \\
18.6 \\
25.4\end{array}$ & 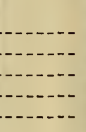 \\
\hline $\begin{array}{l}50 \\
60 \\
66-\ldots\end{array}$ & $\begin{array}{l}17.3 \\
17.5\end{array}$ & $\begin{array}{l}21.0 \\
25.0\end{array}$ & $\cdots$ & $\begin{array}{l}36.3 \\
38.6\end{array}$ & $\begin{array}{r}16.8 \\
18.0\end{array}$ & $\begin{array}{r}9.2 \\
11.8\end{array}$ & $\begin{array}{l}38.5 \\
59.5 \\
75.5\end{array}$ & \\
\hline $\begin{array}{l}70- \\
72\end{array}$ & 17.7 & $\begin{array}{l}30.0 \\
31.2\end{array}$ & & 41.3 & 19.2 & 15.5 & & 77.0 \\
\hline $\begin{array}{l}80 \\
90 \\
100\end{array}$ & $\begin{array}{l}18.0 \\
18.2 \\
18.4\end{array}$ & $\begin{array}{c}31.2 \\
-\cdot \\
-\end{array}$ & $\begin{array}{l}31.9 \\
32.9 \\
34.4\end{array}$ & $\begin{array}{l}44.2 \\
47.6 \\
51.3\end{array}$ & $\begin{array}{l}20.6 \\
22.2 \\
24.0\end{array}$ & $\begin{array}{l}21.2 \\
28.8 \\
38\end{array}$ & & $\begin{array}{l}80.5 \\
85.2 \\
94.6\end{array}$ \\
\hline
\end{tabular}

\section{DISCUSSION OF RESULTS}

In the present series of experiments duplicates usually checked within $0.05 \mathrm{~g}$ of anhydrous salt per $100 \mathrm{~g}$ of water, and determinations approaching equilibrium from saturation conditions on either side located the desired points within $0.2 \mathrm{~g}$ in nearly all cases. Since the temperature was closely controlled and accurately known, it is believed that the determinations for all the salts except magnesium formate are accurate to $\pm 0.1 \mathrm{~g}$ of anhydrous salt per $100 \mathrm{~g}$ of water. These same limits hold in the upper range for magnesium formate, but not below $40^{\circ} \mathrm{C}$. Because of corroborating evidence it is thought, however, that these latter values are accurate within $\pm 0.25 \mathrm{~g}$ per $100 \mathrm{~g}$ water.

The agreement of the values found for calcium formate with previous closely concordant values indicates that the method and procedure are capable of yielding accurate results.

The data on the solubility of strontium formate given by Stanley (12) contain an incorrect factor for converting strontium sulphate to formate, which results in low values throughout his table. ${ }^{3}$ The corrected values are given in figure 4. Information concerning the details of the investigation is very meager. No other investigator has confirmed the existence of the monohydrate, which Stanley gives (without offering proof of its composition) as the form in equilibrium above $84^{\circ} \mathrm{C}$. In the present investigation only the anhydrous salt was found in this temperature range.

Stanley's results for barium formate cannot be better evaluated than those for strontium formate. His table of data includes arithmetical errors, and his value for barium as sulphate is 0.64 percent below the theoretical; values plotted in figure 5 have been corrected for the former effect. The values given by Krasnicki (13) indicate, by the shape of the curve obtained, that his apparatus became increasingly inadequate as its temperature departed from that of the room. The apparatus consisted of an adaptation of a large air bath used by a

${ }^{3}$ Corrected values are given in A. Seidell, Solubilities of Inorganic and Organic Compounds, 2d ed., p. 681, D. Van Nostrand Co., New York, 1919. 
previous investigator (27), and was apparently incapable of maintaining the necessary temperatures. The work of other investigators (14) (28) on the solubility of some of the salts which Krasnicki investigated, and on similar salts, indicates a systematic error in the work of the latter investigator.

\section{TRANSITION POINTS}

In the case of both strontium and cadmium formates the dihydrate in equilibrium with saturated solution at room temperatures is replaced by the anhydrous salt at a higher temperature. The point of transition in each of these cases is determined in the present investigation from the break obtained in the solubility curve.

This transition was found to occur for strontium formate at $72.0^{\circ}$ to $72.5^{\circ} \mathrm{C}$. This is not in agreement with the findings of Stanley (12), but is closely concordant with the temperature $71.4^{\circ}$ to $72.4^{\circ} \mathrm{C}$. found by Plathan (6) using a dilatometric method. The transition for cadmium formate occurs at $66.0^{\circ}$ to $66.5^{\circ} \mathrm{C}$. No previous value was found.

\section{HARRIS'S ADAPTATION OF DÜHRING'S RELATION}

It has recently been shown by R. L. Harris (29) that Dühring's empirical relation for vapor pressures may be adapted to give a relation for the solubilities of anhydrous inorganic salts. Dühring showed that, if the temperatures at which a substance exhibits known vapor pressures are plotted against the temperatures at which a second substance has the same vapor pressures, a straight line is obtained. Harris adapted this by substituting molal concentration for vapor pressure, and obtained a similar relation.

This does not hold true for hydrated salts in many cases, as Harris pointed out, though some do give a series of lines with intersections at transition points. Many hydrates give a straight line until they approach a transition and then swing off in a curve. Other hydrates, and some organic salts, show a regular curve instead of a straight line.

Since the salts here investigated show both the hydrated and anhydrous forms, and since they may be considered as being on the border line between organic and inorganic salts, it was decided to apply the modified Dühring relation to them. A reference salt with comparable solubility was found in cesium chlorate (30).

The results of the application are shown in figure 8 , from which it may be seen that all the anhydrous forms occurring in the series show the straight-line relation. The curve for anhydrous cadmium formate is plotted using extrapolated values for the reference salt.

Strontium formate dihydrate shows the exception noted by Harris of giving a line, straight in the lower portion of the range, and swinging off into a curve as it approaches the transition point. The dihydrates of zinc and cadmium formates show curves over their entire ranges.

The values for magnesium formate dihydrate, taken from the solubility curve obtained by joining the definite upper portion to the definite eutectic point, give a straight line. This corroborates the values obtained for the solubility of magnesium formate below $40^{\circ} \mathrm{C}$. 


\section{ACKNOWLEDGMENT}

Quantities of a number of metallic formates, of which several were used in this investigation, were kindly supplied by Dr. F. R. von Bichowsky, of the United States Naval Research Laboratory. This assistance is gratefully acknowledged.

\section{BIBLIOGRAPHY}

1. E. S. Larsen, The Microscopic Determination of the Nonopaque Minerals, U.S. Geological Survey Bull. 679, Government Printing Office, Washington, 1921.

2. (a) A. N. Winchell, The Microscopic Characters of Artificial Solid Substances or Artificial Minerals, J. Wiley \& Sons, Inc., New York, 1931.

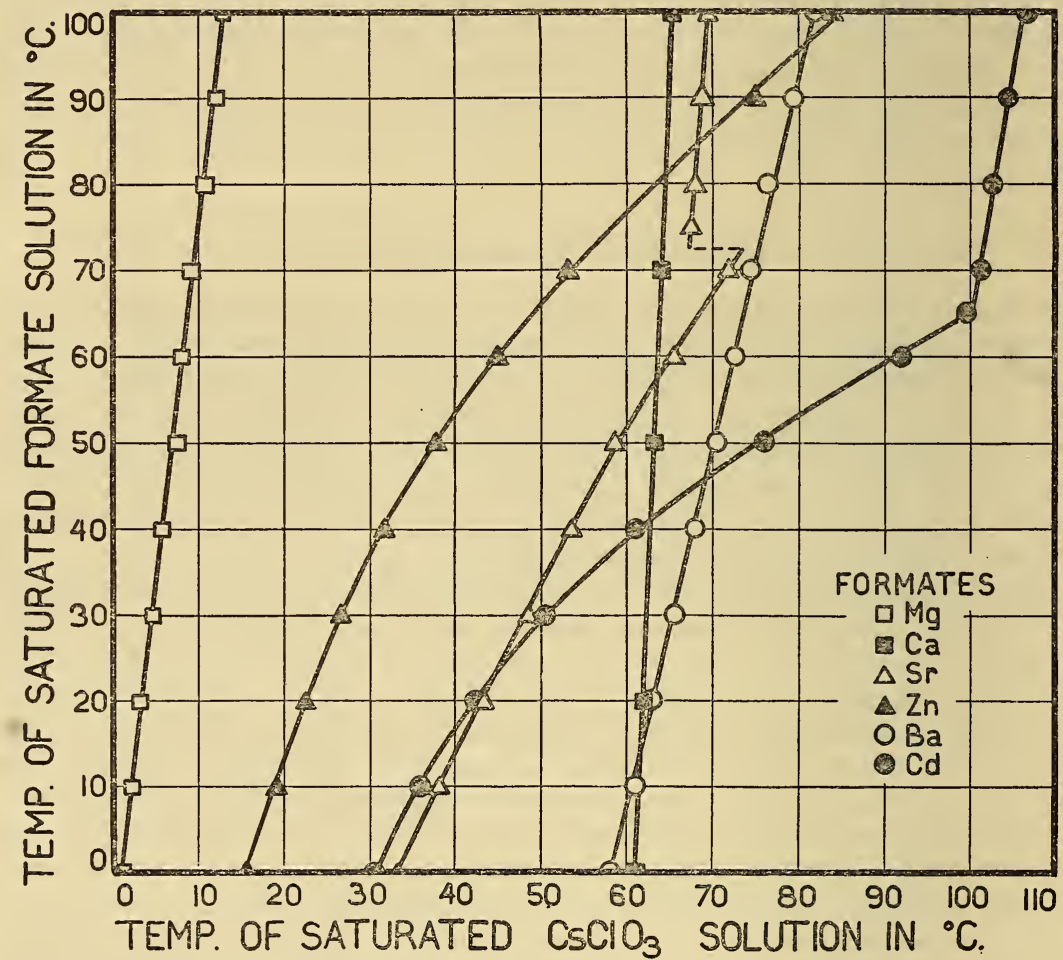

FIgURE 8.-Modified Dühring's relations of formates.

(b) P. Groth, Chemische Krystallographie, 5 vols., Wilhelm Engelmann, Leipzig, 1906-1919.

(c) International Critical Tables, vol. 1, pp. 165-174, McGraw-Hill Book Co., New York, 1926.

3. (a) W. M. D. Bryant, J. Am. Chem. Soc., vol. 54, p. 3758, 1932.

(b) J. B. Wilson and G. L. Keenan, J. Assoc. Official Agr. Chem., vol. 13, p. $389,1930$.

(c) M. Phillips and G. L. Keenan, J.Am.Chem.Soc., vol. 53, p. 1924, 1931.

(d) V. H. Morris, J.Am.Chem.Soc., vol. 54, p. 2843, 1932.

(e) C. F. Poe and J. E. Sellers, J.Am.Chem.Soc., vol. 54, p. 249, 1932.

(f) International Critical Tables, vol. 1, pp. 278-280, McGraw-Hill Book Co., New York, 1926.

4. P. Groth, Chemische Krystallographie, vol. 3, pp. 7 et seq., Wilhelm Engelmann, Leipzig, 1910. 
5. A. Schrauf, Sitzber. Akad. Wiss. Wien, Math. naturw. Klasse, vol. 42, p. 125,1860 .

6. (a) A. Plathan, Ueber eine isomorphe Reihe von Formiaten des Calcium, Strontium, Baryum und Blei, p. 16, O. W. Bachmans Buchdruckerei, Kuopio, 1897.

(b) A. Plathan, Dissertation, Helsingfors, 1900. (An extended study of the same material found in $6 a$.)

7. (a) H. Schröder, Ber., vol. 8, p. 199, 1875.

(b) H. Schröder, Ber., vol. 14, p. 21, 1881.

8. F. W. Clarke, Am. Chem.J., vol. 2, p. 174, 1880-81.

9. L. Wagner, Z. Krist., vol. 50, p. 49, 1912.

10. P. Alexatt, Bull. Soc. Imp. Naturalistes Moscou, 1897, pp. 446-468, 1898. From abstract in Z. Krist., vol. 32, p. 504, 1900.

11. I. Nitta, Sci. Papers Inst. Phys. Chem. Research (Tokyo), vol. 9, p. 151, 1928.

12. H. Stanley, Chem. News, vol. 89, p. 193, 1904.

13. E. v. Krasnicki, Monatsh., vol. 8, p. 595, 1887.

14. J. S. Lumsden, J.Chem.Soc., vol. 81, p. 350, 1902.

15. A. Souchay and C. Grolle, J. prakt. Chem., vol. 76, p. 474, 1859.

16. W. F. Hillebrand and G. E. F. Lundell, Applied Inorganic Analysis, p. 490, J. Wiley \& Sons, Inc., New York, 1929.

17. Ibid., p. 491 .

18. Ibid., p. 492.

19. Ibid., p. 503.

20. Ibid., footnote, p. 493.

21. T. W. Richards, L. P. Hall, and B. J. Mair, J.Am.Chem.Soc., vol. 50, p. 3308, 1928.

22. J. Kendall and H. Adler, J.Am.Chem.Soc., vol. 43, p. 1476, 1921.

23. E. W. Dean and D. D. Stark, J.Ind.Eng.Chem., vol. 12, p. 487, 1920.

24. H. Schröder, Ber., vol. 14, p. 23, 1881.

25. H. J. Wing, Ind.Eng. Chem., Anal. ed., vol. 2, p. 196, 1930.

26. P. Calamé, Z. physik. Chem., vol. 27, p. 405, 1898.

27. G. Raupenstrauch, Monatsh., vol. 6, p. 563, 1885.

28. (a) J. Walker and W. Fyffe, J.Chem.Soc., vol. 83, p. 173, 1903.

(b) H. J. Wing and T. J. Thompson, J.Am.Chem.Soc., vol. 48, p. 104, 1926.

(c) H. J. Wing, J.Am.Chem.Soc., vol. 49, p. 2859, 1927.

29. R. L. Harris, Ind.Eng. Chem., vol. 24, p. 455, 1932.

30. International Critical Tables, vol. 4, p. 243, McGraw-Hill Book Co., New York, 1928.

31. R. C. Emmons in A. N. Winchell's Microscopic Characters of Artificial Inorganic Solid Substances, pp. 130-142, J. Wiley \& Sons, Inc., New York, 1931 .

Washington, June 1, 1933. 